\title{
TRANS-
}

Revue de littérature générale et comparée

\section{La Résistance cinématographique à la littérature, des formalistes russes à Dos Passos}

When film resists literature: from Russian formalists to Dos Passos

\section{Nikol Dziub}

revues.org

Éditeur

Presses Sorbonne Nouvelle

Édition électronique

URL : http://trans.revues.org/1254

ISSN : 1778-3887

Référence électronique

Nikol Dziub, « La Résistance cinématographique à la littérature, des formalistes russes à Dos Passos », TRANS- [En ligne], 20 | 2016, mis en ligne le 14 octobre 2016, consulté le 10 novembre 2016. URL: http://trans.revues.org/1254; DOI : 10.4000/trans.1254

Ce document a été généré automatiquement le 10 novembre 2016.

Tous droits réservés 


\title{
La Résistance cinématographique à la littérature, des formalistes russes à Dos Passos
}

When film resists literature: from Russian formalists to Dos Passos

\author{
Nikol Dziub
}

\section{Introduction}

1 Nous voudrions, dans le présent article, étudier la notion de résistance dans le discours théorique littéraire et cinématographique à l'époque du formalisme, et essayer de cerner la portée politique et culturelle de la résistance à la littérature. Nous étudierons tout d'abord la pratique hautement métatextuelle de l'étrangéisation, qui consiste à déconstruire le geste mimétique afin de construire un discours politique. Nous souhaiterions aussi montrer comment le procédé typiquement cinématographique du montage a donné lieu à des tentatives d'imitation en littérature (nous pensons notamment au simultanéisme de Dos Passos). C'est alors le cinéma qui sert de modèle poétique et politique à une littérature qui tente de se résister à elle-même. Enfin, nous tenterons de décrire les stratégies mises en place par Dos Passos pour faire barrage au flot des mots et à la temporalité linéaire de la littérature.

\section{La défamiliarisation}

Dans son article intitulé « ИСКУССТВО КАК ПРИЕМ » («L'Art comme procédé », 1917), le formaliste russe Victor Chklovski introduit le concept d'ОстРАНЕ́нИЕ (défamiliarisation), que Todorov traduit en 1965 par «singularisation ${ }^{1}$ " (mais bien avant que Todorov n'importe le concept, ou plutôt le mot qui le désigne, la théorie littéraire et l'art théâtral français s'en étaient emparés). En quoi consiste la révolution opérée par ce concept? Il 
faut noter d'abord que les formalistes refusent l'approche philosophique, psychologique et sociologique (analyse d'un texte à partir du contexte biographique et social qui fut celui de sa production) propre à la critique littéraire russe de l'époque. C'est peut-être la raison pour laquelle Todorov préfère le terme de «singularisation" à celui de défamiliarisation. En effet, Chklovski défend l'idée d'une littérature ayant ses propres dispositifs et préoccupations, d'une littérature qui se singularise et se distingue ainsi des autres domaines de l'activité humaine. Dziga Vertov reprendra un peu plus tard la même idée, mais en l'appliquant au cinéma. Ce sur quoi Chklovski et Vertov insistent en particulier, c'est sur l'idée de «fabrication». Ainsi, dans L'Homme à la caméra (1929), Vertov introduit des figures autoréflexives, comme celles du tissage et de la roue qui tourne. Le cinéma ne doit pas se contenter de montrer - il doit construire aussi, forger, faire. Dans les films de Vertov, la paresse est très mal vue. S'il insiste tant sur les scènes de travail à l'usine, c'est pour figurer, autoréflexivement, le travail auquel doit se livrer le réalisateur s'il ne veut pas tomber dans une mimésis parasitaire.

Pour en revenir à la notion d'ostranenié, ce terme désigne, d'un point de vue technique, les procédés littéraires qui retardent la transmission du sens. Ce procédé permet de prêter aux mots la résistance qui est celle des choses. L'article de Chklovski commence par l' affirmation suivante : «L'art, c'est la pensée par images », et continue ainsi : «L'art est avant tout créateur de symboles ${ }^{2}$.» Chklovski poursuit en soulignant que cette définition a " résisté et a survécu à l'effondrement de la théorie sur laquelle elle était fondée ${ }^{3}$ ». Puis il définit l'ostranenié et illustre son propos en se référant à un passage de La Guerre et la Paix où Tolstoï, dans un geste proto-déconstructeur et anti-platonicien, invite le lecteur à découvrir une œuvre opératique comme s'il ne savait rien de l'art. Il faut que l'objet d'art soit aussi étranger au récepteur qu'un objet non-artistique : «Le but de l'art, c'est de donner une sensation de l'objet comme vision et non pas comme reconnaissance; le procédé de l'art est le procédé qui consiste à obscurcir la forme, à augmenter la difficulté et la durée de la perception ${ }^{4}$. " De la sorte, le récepteur cesse de trouver naturel ce qui n'est que conventionnel, et toute œuvre d'art apparaît comme un artefact, y compris, par le biais d'un saut autoréflexif, l'œuvre (méta)littéraire qui prétend dénoncer le caractère essentiellement construit de toute représentation. Notons que Brecht se souviendra de sa lecture de Chklovski au moment de développer le concept de "Verfremdung" (étrangéisation - ce qui n'est rien d'autre qu'une traduction allemande du mot ostranenié ) : dans un mouvement dialectique, il prétend ne jamais montrer un personnage ou un fait qu'en le rendant immédiatement insolite.

\section{Du pur cinéma}

4 La résistance est l'action d'une force qui s'oppose à une autre force, la résistance est un blocage, elle est aussi refus de l'hétérogénéité. L'article de Chklovski ouvre des pistes non seulement aux théoriciens, mais aussi aux cinéastes (notamment à Dziga Vertov et à Eisenstein). Dans son manifeste intitulé Ciné-đEil, publié en 1923 dans le troisième numéro de la revue ЛЕФ : ЛЕВЫЙ ФРОнТ искусстВ ${ }^{5}$, éditée par Maïakovski, Vertov prétend faire du cinéma un instrument de résistance au passé, notamment littéraire. Si toute œuvre littéraire se construit sur d'énormes épaisseurs de littérature (Antoine Compagnon, citant Barthes, note que, "[s]ans origine, "le texte est un tissu de citations": la notion d'intertextualité se dégage elle aussi de la mort de l'auteur $\left.{ }^{6} »\right)$, l'œuvre cinématographique ne peut se tourner que vers elle-même. La littérature apparaît de plus 
comme une institution de pouvoir, dont la grammaire (ou la poétique, si l'on préfère) est un instrument de contrainte. Refuser l'adaptation littéraire, rejeter tout sujet, toute intrigue, tout scénario, c'est dès lors faire acte de résistance aussi bien artistique que morale et épistémologique. (Les hommes de littérature, d'ailleurs, s'en prendront aux formalistes : en témoigne la charge de Gorki intitulée « Sur le formalisme » et publiée en $\left.1936^{7}\right)$.

5 Le générique du film L'Homme à la caméra (1929) est suivi d'un sous-titre hautement métatextuel: «extrait du journal du ciné-opérateur ${ }^{8}$ " (le film prétend être autobiographique). Puis vient un avertissement au lecteur, qui présente le film comme une construction: "Attention, spectateur: le vrai film représente en soi l'expérimentation d'une ciné-émission de phénomènes visibles ${ }^{9}$.» Vertov annonce ensuite à quoi son cinéma résistera. D'abord, il sera indépendant des mots et du langage, l'image n'aura plus besoin du soutien des inscriptions écrites ${ }^{10}$. Ensuite, le cinéma sera indépendant de la littérature. Les images seront convaincantes sans l'aide du scénario ${ }^{11}$. Enfin, le cinéma sera indépendant du théâtre, et renoncera aux décors ${ }^{12}$. À la fin de ce générique-préface, Vertov écrit : « Ce travail expérimental est destiné à créer le véritable langage international absolu du cinéma, qui sera tout à fait distinct du langage du théâtre et de la littérature ${ }^{13}$.»

6 La première image montre une caméra, si grande que l'homme à la caméra se tient dessus 14. Puis le film montre un ancien théâtre devenu cinéma, qui attire un public nombreux ${ }^{15}$. Chaque image contredit la précédente, selon une logique du contraste (luxe/pauvreté, bourgeoisie/peuple, paresse/travail, irresponsabilité/responsabilité). L'œil de la caméra montre ce que le spectateur ne verrait pas seul. Mais surtout, il construit sa vision : il peut répéter une image, il peut jouer sur la géométrie des formes, sur les contrastes. Vertov filme aussi, dans un geste métadiscursif, le changement d'objectif ${ }^{16}$. Le téléobjectif, par exemple, permet de se rapprocher des objets et des personnes. Vertov montre aussi comment fonctionne l'ouverture de l'objectif, et comment l'homme à la caméra cligne de l'objectif comme on cligne de l'œil ${ }^{17}$. Plusieurs fois, on voit une affiche portant le slogan suivant : « Réveil ». Le cinéma doit réveiller le spectateur, lui ouvrir les yeux.

7 Le discours d'Eisenstein sur le cinéma ${ }^{18}$ n'est toutefois pas si éloigné de celui de Vertov. Il ne se montre pas beaucoup plus amical que Vertov à l'égard du cinéma littéraire, même s'il accepte d'entrer en dialogue avec une œuvre dont il considère qu'elle s'apparente à un suicide de la littérature : Ulysses (à propos de l'œuvre de Joyce, Eisenstein affirmait que la littérature sortait irrémédiablement de ses limites en tentant d'accomplir les possibilités du monologue intérieur). Dans une lettre datée du 15 février 1928, il exprime sa joie d'avoir reçu le déjà célèbre «roman », paru en 1922 : «Reçu Ulysse samedi, la bible du nouveau cinéma ${ }^{19}$ ».

8 Par ailleurs, Eisenstein défend une poétique dialectique, qui, mutatis mutandis, est celle de Vertov. Ce qu'il propose de construire, c'est ce que Brecht appellera un voir complexe. Pour Eisenstein, le montage est un langage symbolique (voir l'article intitulé «диккенс, гРИФфит и мы », « Dickens, Griffith et Nous ») et défend l'idée que le montage n'est pas l'œuvre de l'auteur, mais le résultat d'un travail collectif ${ }^{20}$. Eisenstein fait régulièrement allusion aux thèses marxistes, bien sûr, mais aussi à la Révolution française de 1789 et à Napoléon. Le film intitulé Octobre (1927) est particulièrement intéressant à cet égard. Eisenstein s'y inspire du livre de John Reed, Dix jours qui ébranlèrent le monde (1920). Tout en proposant un discours sur la révolution et en développant son art du montage, Eisenstein ne peut s'empêcher d'introduire dans son film des éléments littéraires, et de 
mêler, pour reprendre la terminologie brechtienne, des "éléments formulés » aux " éléments formels ${ }^{21}$ ».

\section{Ce que le montage fait à la littérature}

Octobre sert en quelque sorte de pont entre littérature et cinéma. Le film influencera considérablement John Dos Passos. Ce dernier séjourne en Russie en 1928. Il veut découvrir ce système d'Etat, cette alternative au capitalisme américain, mais aussi étudier le théâtre d'avant-garde russe, alors à son apogée. Dos Passos rencontre Eisenstein, et s'intéresse à son «principe de montage général ». Le principe de la polyphonie leur est commun, comme le refus de la figure de l'auteur. Ainsi, si un certain cinéma résiste à la littérature, les écrivains, eux, se laissent influencer par le cinéma. Dos Passos (dans U.S.A., mais aussi dans les textes qu'il écrit en vue de promouvoir son "Théâtre révolutionnaire ${ }^{22}$ ») développe en littérature la technique du "camera eye » de Vertov, dans le but de garantir au lecteur la neutralité d'une écriture a-focalisée. Ce faisant, il renonce à deux des instruments les plus importants de l'institution littérature: le discours et l'auteur (l'auteur, selon la nouvelle critique française, et en particulier selon Barthes, qui annonce en 1968 la «mort de l'auteur», n'étant "autre que le bourgeois, l'incarnation quintessentielle de l'idéologie capitaliste $\left.{ }^{23} »\right)$.

La résistance cinématographique à la littérature est donc loin d'être toute négative. Les formalistes dits russes (qui publièrent souvent dans des revues ukrainiennes ou tchécoslovaques) et Dos Passos remplacent en effet le discours d'auteur par un instrument nouveau: le montage de l'œuvre. Ce qui est important dans la prose de Dos Passos c'est la forme - une forme qui résiste à la notion traditionnelle de forme. Ce refus de la règle est essentiellement politique : « individualisme et contestation du pouvoir sous toutes ses formes [...] l'avaient amené dès One Man's Initiation (1917) à prôner, plus ou moins ouvertement, une certaine forme d'anarchie ${ }^{24} »$. Dos Passos tente de développer une logique de la forme « communiste ». D'ailleurs, l'absence de ponctuation, les phrases inachevées, pour Chklovski, relèvent d'un " processus d'automatisation ${ }^{25}$ » anti-auctorial. À la démarche essentiellement digressive du discours auctorial (il s'agit toujours de tourner autour du fait, de l'envelopper dans une œuvre-artefact qui le voile et le dénature - signalons à ce propos que la Verfremdung brechtienne n'est qu'un antidote à l' Entfremdung, à l'aliénation) se substitue une démarche constructive : l'œuvre, qui se veut objective aussi bien dans sa genèse que dans son propos, se monte, s'érige, se construit elle-même, hors de tout sujet produisant ou recevant.

Le montage est une pratique, c'est un acte matériel et idéologique, c'est un acte (d'interprétation de la réalité) politique : c'est pour cela qu'Eisenstein parle du montage comme d'un langage. Félicie Pastorello souligne que «[1]e débat esthétique sur le montage s'ouvre [...], en dernière instance, sur le politique ${ }^{26} \%$. Le cinéma est un puissant instrument de propagande. Il suffit, pour s'en convaincre, de se souvenir de la phrase de Lénine : «le cinéma est pour nous, de tous les arts, le plus important ». Mais il n'y pas de politique sans poétique.

Pour les formalistes, la forme est l'essentiel dans l'art, mais ils insistent aussi sur l'idée que la fabula n'est pas nécessaire. Ils prennent pour fondement de leur pensée les distinctions génériques littéraires. Le cinéma formaliste russe repose tout entier sur le rejet de la fabula. Pour Chklovski en particulier, la distinction cinéma de prose/cinéma de 
poésie se superpose à la distinction fable/composition. Dans l'ouvrage intitulé La Poétique du cinéma (1927), dirigé par Boris Eikhenbaum, le groupe des formalistes tente de poser les fondements théoriques du cinéma (en tant qu'art ou médium de communication, avec sa stylistique et ses procédés propres, mais aussi dans ses rapports à l'art pictural, au théâtre et à la littérature). Le terme "poétique " est bien employé dans le sens que lui donne Aristote, mais les formalistes opèrent une sorte de syncrétisme entre poétique et poésie. Dans le "cinéma de poésie ", il y a plus d'éléments formels que d'éléments de sens. "Le cinéma sans sujet - est le cinéma de poésie ${ }^{27}$ » (dans le texte original, il y a des guillemets autour de "poésie ») - telle est la dernière phrase de la contribution de Chklovski dans l'ouvrage cité.

\section{Ce qui résiste aux mots}

13 Brecht également fait partie de ces écrivains qui tentèrent de résister à la littérature. Dans un texte inspiré de Chklovski, «Sur la distanciation dans le jeu» (1936), Brecht propose au théatre occidental de prendre pour exemple un certain théâtre chinois qui affiche, selon le principe à la fois dialectique et métatextuel du Verfremdungseffekte, le dédoublement du comédien, qui est à la fois lui-même et son personnage. Dix ans plus tôt déjà, Brecht (qui se voulait l'ennemi des «théâtres [qui] se moquent que les jeunes gens ne viennent pas, car ceux-ci n'ont pas d'argent ${ }^{28}$ ») annonçait : «Le Théâtre est mort ${ }^{29}$ » (1936). «L'art est-il encore possible ?30 » se demandait celui qui « commençait presque à douter du théâtre et de l'art en général ».

La décadence du théâtre occidental irrite de la même manière Dos Passos. Les voyages de Dos Passos hors Amérique sont aussi des fuites loin de la littérature américaine. Dans "Against American Literature", il critique la tendance américaine (initiée par Washington Irving) à la satire. «Our books are like our cities; they are all the same ${ }^{31}$ », note-til également. Dos Passos préfère la littérature et le théâtre russes, précisément parce qu'ils mettent le lecteur ou le spectateur au contact de choses bien palpables, qui résistent aux mots : "The Russian stirs eyes and ears and nose and sense of touch, portrays his story with vivid tangibility; the American leaves an abstract impression of intellectual bitterness ${ }^{32}$." Dos Passos est en particulier fasciné par Vsevolod Meyerhold - "architecte de scène ", mentor d'Eisenstein, premier responsable du théâtre des Soviets, avant d'être accusé de trotskisme et d'être exécuté sur ordre de Staline en 1940. Dans un article intitulé « Is the " Realistic" Theatre Obsolete? ", paru dans Vanity Fair en 1925, Dos Passos se met par ailleurs à la place d'un Man-on-the-Street qui n'as pas accès, intellectuellement parlant, à ce que propose le théâtre américain - alors même que «since religion has failed humanity, the theatre is the focus of mass emotion ${ }^{33}$ ». L'existence du cinéma lui est un argument pour résister à cette institution littéraire obsolète - le théâtre :

The movies have made the theatre of the transparent fourth wall unnecessary and obsolete, just as photography has made obsolete a certain sort of painting. The camera and screen can transport the audience into circumstances, in the ordinary sense, real ${ }^{34}$.

Il est d'ailleurs curieux de noter que, si le montage a été théorisé par les cinéastes soviétiques, qui ont influencé Dos Passos, aux yeux de Deleuze, par exemple, il est propre à la culture américaine. Et de fait, les premiers textes d'Eisenstein sur le montage se fondaient sur le film de Griffith, Birth of a Nation (1915). Sans compter que Dos Passos, écrivain américain malgré lui, est le fondateur, sinon du simultanéisme littéraire, du moins d'un certain simultanéisme américain. 
16 En 1938, Sartre consacre un article au 1919 de Dos Passos. Il admire Dos Passos parce qu'il est capable de rendre « la singularité d'une vie ${ }^{35}$ ». Et précisément, quand il entreprend de construire un personnage résistant, résistant à l'entraînement horizontal du texte, Dos Passos fait allusion au cinéma - au cinéma quotidien :

Then it would be a great relief to get out of the stuffy house and the stuffy treeshaded streets of Georgetown and to stop by for Alice Dick and go downtown to the moving pictures and to see the pictures of foreign countries, and the crowds on F Street and to stop in at a drugstore for a soda afterwards, before getting on the Georgetown car, and to sit up at the fountain talking about the picture they'd seen and Olive Thomas and Charley Chaplin and John Bunny. She began to read the paper every day and to take an interest in politics ${ }^{36}$.

Mais Sartre note encore: "Le monde de Dos Passos est impossible - comme celui de Faulkner, de Kafka, de Stendhal - parce qu'il est contradictoire. Mais c'est pour cela qu'il est beau : la beauté est une contradiction voilée. Je tiens Dos Passos pour le plus grand écrivain de notre temps ${ }^{37}$.» On peut supposer que Sartre fait allusion au simultanéisme développé par Dos Passos, qui consiste à juxtaposer des éléments selon une logique tabulaire, et non à les relier selon une logique linéaire. C'est la forme que prend, en littérature, le principe cinématographique d'alternance dialectique. Sartre compare Dos Passos aux maitres de la technique du self-consciousness et à ceux qui ont remis en cause les pratiques littéraires traditionnelles du temps: «La plupart des grands auteurs contemporains, Proust, Joyce, Dos Passos, Faulkner, Gide, V. Woolf, chacun à sa manière, ont tenté de mutiler le temps ${ }^{38}$. » Le cinéma mutile le temps littéraire, il le lacère, il le fragmente, il s'attaque à son illusoire continuité. Dos Passos, incontestablement, utilise des techniques qui empêchent la narration traditionnelle de se développer, il place une scène à côté d'une autre, en les juxtaposant sans se soucier du développement chronologique de l'histoire. Certes, dans son Esthétique du montage, Vincent Amiel, avance cette idée que «la simultanéité exprimée par le montage alterné n'est en fait qu'une excroissance, une utilisation marginale de la linéarité de l'action suggérée par le montage $c^{2} t^{39} »$. Mais il n'en demeure pas moins « la simultanéité est une anomalie par rapport à la succession temporelle, dans laquelle tout récit place forcément deux événements qu'il rapporte l'un après l'autre ${ }^{40} »$.

Dans U.S.A., et plus précisément dans la section intitulée "Camera Eye ", Dos Passos supprime la ponctuation, et joue, typographiquement, sur les espaces, parfois trop longues, parfois presque inexistantes. Le lecteur a le sentiment lire, et presque de voir, une série de séquences, et il lui semble que c'est son rôle de repérer les liens possibles, de construire les connexions. Il n'y a plus de diégèse (cette entité fluide qui est abandon), et plus de temps diégétique. L'écriture de la simultanéité est un acte de résistance au temps littéraire, et suppose l'élaboration d'une architecture de l'instant. À en croire Deleuze, dans le cinéma, il ne faut pas interroger la diégèse, mais la vision. Il est certain que la caméra a instauré une relation nouvelle avec le regard comme avec le temps, relation praticable aussi bien en littérature qu'au cinéma. Le simultanéisme, d'ailleurs, est aussi un internationalisme verbal. Les mots étrangers sont très nombreux, dans U.S.A., mais ils ne sont pas mis en italiques. Dos Passos construit la résistance des mots dans son œuvre. Ce sont des mots réels, originaux, irréductibles, qui n'ont rien de naturel, qui font obstacle à la lecture, qui résistent au lecteur. Dos Passos n'hésite pas par ailleurs à recourir au collage, et transforme parfois son livre en journal (voir des sections « Newsreel »). Il résiste ainsi au désengagement essentiel de la littérature - désengagement qui explique la tendance du récit littéraire à aller toujours de l'avant, comme si rien ne pouvait l'arrêter, comme si rien ne pouvait lui résister. Ce combat sera-t-il couronné de 
succès ? La dernière des cinquante-et-une séquences « Camera-Eye[s] », dans U.S.A., permet d'en douter, puisqu'elle dénonce la coupable légèreté de toute littérature, aussi résistante soit-elle :

He lifts his hand towards the telephone

The deputies crowd in the door

We have only words against ${ }^{41}$

\section{Conclusion}

Il faut donc tenter de résister à la littérature en tant qu'elle est une construction, institutionnelle comme poétique, qui se prétend naturelle. C'est contre ce double geste de construction et de dissimulation que luttent ceux - cinématographes ou écrivains - qui pratiquent la défamiliarisation (essentiellement critique) et le montage (constitutivement pratique). Reste que, si les cinématographes peuvent se passer des outils de l'institution littéraire, les écrivains n'ont d'autre choix que de composer (dans tous les sens du terme) avec les mots. L'art (ou l'artifice) du montage permet alors de faire barrage à l'écoulement faussement fatal du flux verbal.

\section{BIBLIOGRAPHIE}

AMENGUAL (Barthélemy), ¡Que viva Eisenstein !, Lausanne, L’Âge d'Homme, 1980.

AMIEL (Vincent), Esthétique du montage, 3è éd., Paris, Armand Colin, 2014.

BLeU-SCHWENninger (Patricia), John Dos Passos, l'écriture-miroir, Grenoble, ELLUG, 1993.

BOUQUET (Stéphane), Sergueï Eisenstein, Paris, Cahiers du cinéma, coll. « Grands Cinéastes », 2007.

BRECHT (Bertolt), « Sur le théâtre que nous avons en tête. Notre public », in BRECHT (B.) Écrits sur le théâtre, éd. J.-M. Valentin, Paris, Gallimard, L'Arche, coll. « Bibliothèque de la Pléiade », 2000.

CHKLOVSKI (Victor), « L'Art comme procédé », in TODOROV (T.), Théorie de la littérature. Textes de formalistes russes. Réunis, présentés et traduits par T. Todorov, avec une préface de R. Jakobson, Paris, Éd. du Seuil, 1965.

-- « Poésie et prose dans l'art cinématographique », in ALBERA (F.) (dir.) Les Formalistes russes et le cinéma. Poétique du film, introduit et commenté par F. Albéra, trad. du russe par V. Posener, R. Gayraud et J.-Ch. Peuch, Paris, Nathan, 1996.

COMPAGNON (Antoine), Le Démon de la théorie. Littérature et sens commun, Paris, Éd. du Seuil, 1998.

Deleuze (Gilles), Cinéma 1. L'Image-mouvement, Paris, Minuit, coll. « Critique », 1983.

DOS PASSOS (John), U.S.A., Middlesex, Penguin Books, 1973.

-- U.S.A., Paris, Gallimard, 2002.

-- Travel Books and Other Writings 1916-1941, New York, The Library of America, 2003. 
EISENSTEIN (Sergueï Mikhaïlovitch), « Sur la question d'une approche matérialiste de la forme » (1925), Cahiers du cinéma, nº 220-221, mai-juin 1970.

MOREL (Jean-Pierre), « Jules Romains et Dos Passos. Remarques », in VIART (D.) (éd.), Jules Romains et les écritures de la simultanéité, Villeneuve d'Ascq, Presses Universitaires du Septentrion, coll. «travaux et recherches », 1996, p. 223-236.

PAStorello (Félicie), « La Catégorie du montage chez Tretjakov, Arvatov, Brecht », in BABLET (D.) (dir.), Collage et montage au théâtre et dans les autres arts durant les années vingt, Lausanne, L'Âge d'Homme, 1978, p. 117-128.

SARTRE (Jean-Paul), « À propos de John Dos Pasos et de 1919 », Situations, I, Paris, Gallimard, 1947.

\section{NOTES}

1. Victor Chklovski, «L'Art comme procédé », in Théorie de la littérature. Textes de formalistes russes réunis, présentés et traduits par Tzvetan Todorov, avec une préface de Roman Jakobson, Paris, Seuil, 1965, p. 84 et suivantes.

2. Ibid., p. 76.

3. Ibid., p. 77.

4. Ibid., p. 83.

5. Lef, Levyi Front Iskusstv. Je traduis : « Front gauche des Arts ».

6. Antoine Compagnon, Le Démon de la théorie. Littérature et sens commun, Paris, Seuil, 1998, p. 56.

7. Cet article a été publié pour la première fois dans le numéro 99 de la revue ПРАВДА (Vérité), le 9 avril 1936.

8. Je traduis. Voir Dziga Vertov, (1929), L’Homme à la caméra, $00: 00: 12 / 01: 08: 44$, vidéo en ligne : https://www.youtube.com/watch?v=z97Pa0ICpn8

9. Je traduis. Voir Ibid., $00: 00: 20 / 01: 08: 44$

10. Je traduis. Voir Ibid., $00: 00: 33 / 01: 08: 44$

11. Je traduis. Voir Ibid., $00: 00: 40 / 01: 08: 44$

12. Je traduis. Voir Ibid., $00: 00: 46 / 01: 08: 44$

13. Je traduis. Voir Ibid., $00: 55 / 01: 08: 44$

14. Voir Ibid., $00: 01: 35 / 01: 08: 44$

15. Voir Ibid., $00: 02: 56 / 01: 08: 44$

16. Voir Ibid., $00: 10: 20 / 01: 08: 44$

17. Voir Ibid., $00: 12: 00 / 01: 08: 44$

18. On pourra se référer à l'ouvrage de Victor Chklovski intitulé Eisenstein, paru en 1973 à Moscou (Iskousstvo, coll. « La vie dans l'art »).

19. Stéphane Bouquet, Sergueï Eisenstein, Paris, Cahiers du cinéma, coll. "Grands Cinéastes », 2007, p. 52.

20. Voir, p. 274-280 du tome 2 des Euvres choisies de S. M. Eisenstein, Moscou, Iskousstvo, 1964, « O ПОЗИЦИИ БЕЛА БАЛАША » (« Sur la position de Béla Balázs ») et « БЕЛА ЗАБЫВАЕТ НОЖНИЦЫ » («Béla oublie les ciseaux »), deux textes parus initialement dans кино (Кino), Moscou, le 20 juin et le 10 août 1926.

21. Cité dans Barthélemy Amengual, ¡Que viva Eisenstein!, Lausanne, L'Age d'Homme, 1980, p. 403.

22. John Dos Passos est l'auteur d'un article intitulé "Toward a Revolutionary Theatre ", paru dans la revue New Masses, 3 (1927).

23. Antoine Compagnon, op. cit., p. 55.

24. Patricia Bleu-Schwenninger, John Dos Passos, l'écriture-miroir, Grenoble, ELLUG, 1993, p. 238.

25. Victor Chklovski, « L’Art comme procédé », op. cit., p. 82. 
26. Félicie Pastorello, «La Catégorie du montage chez Tretjakov, Arvatov, Brecht », in Denis Bablet (dir.), Collage et montage au théâtre et dans les autres arts durant les années vingt, Lausanne, L'Age d'Homme, 1978, p. 127.

27. Victor Chklovski, «Poésie et prose dans l'art cinématographique », in Les Formalistes russes et le cinéma. Poétique du film, introduit et commenté par François Albéra, traduit du russe par Valérie Posener, Régis Gayraud, Jean-Christophe Peuch, Paris, Nathan, 1996, p. 141.

28. Bertolt Brecht, "Sur le théâtre que nous avons en tête. Notre public », in Écrits sur le théâtre, Paris, Bibliothèque de la Pléiade, 2000, p. 67.

29. Ibid., p. 72.

30. Ibid., p. 75.

31. John Dos Passos, « Against American Literature " (publié dans The New Republic, le 14 octobre 1916), in Travel books and other writings 1916-1941, New York, The Library of America, 2003, p. 587.

32. Ibid., p. 589-590.

33. Ibid., p. 592.

34. Ibid., p. 594.

35. Jean-Paul Sartre, «À propos de John Dos Pasos et de 1919 », Situations I, Paris, Gallimard, 1947, p. 22.

36. John Dos Passos, U.S.A., Middlesex, Penguin Books, 1973, p.134. Traduction française de Norbert Guterman (John Dos Passos, U.S.A., Paris, Gallimard, 2002, p. 163) : « Après quoi, c'était un grand soulagement de sortir de la maison étouffante et des rues pleines d'ombre et étouffantes de Georgetown et de passer prendre Alice Dick et de descendre en ville pour aller au cinéma où l'on voyait des films de pays étrangers, de se promener dans la foule de F Street, d'entrer dans un drugstore prendre un soda avant de monter sur le tram de Georgetown et de rester assises devant le comptoir en bavardant sur le film qu'elles venaient de voir et sur olive Thomas et Charlie Chaplin et John Bunny. Elle se mit à lire le journal tous les jours et commença à s'intéresser à la politique. "

37. Jean-Paul Sartre, op. cit., p. 24-25.

38. Ibid.

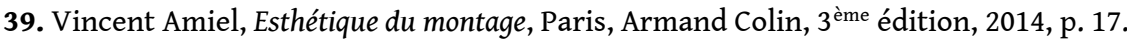

40. Jean-Pierre Morel, «Jules Romains et Dos Passos. Remarques », in Dominique Viart (éd.), Jules Romains et les écritures de la simultanéité, Villeneuve d'Ascq, Presses Universitaires du Septentrion, coll. « Travaux et recherches », 1996, p. 233.

41. John Dos Passos, "The Camera Eye (5)», U.S.A., Middlesex, Penguin Books, 1973, p. 1135. Traduction française de Norbert Guterman, p. 1202 :

il lève sa main vers le téléphone

les flics encerclent la porte

contre qui nous n'avons que des mots

\section{RÉSUMÉS}

Afin que l'art n'apparaisse plus comme naturel, les cinéastes formalistes russes développent un procédé que Chklovski nomme остРАнÉHИE (défamiliarisation). Dans le même temps, ils rejettent le passé littéraire, et prétendent pratiquer un cinéma pur de tout contact avec les autres arts. Vertov puis Eisenstein développent une poétique anti-littéraire du montage, que Brecht et Dos 
Passos tenteront d'exploiter verbalement, afin de résister à la tendance naturelle de la littérature au désengagement.

Art's productions are not natural productions. That's why Russian formalist filmmakers develop a process that Shklovsky calls ОСТРАНЕНИЕ (defamiliarization). At the same time, they reject the literary past : cinema has to be independent of the other arts. Vertov and Eisenstein develop an anti-literay poetics of the montage, that Brecht and Dos Passos try to use, in order to resist literature's disengagement from politics.

\section{INDEX}

Mots-clés : Formalisme, défamiliarisation, résistance, montage, cinéma

Keywords : Formalism, defamiliarization

\section{AUTEUR}

\section{NIKOL DZIUB}

Docteure en littérature générale et comparée (UHA, ILLE), Nikol Dziub enseigne la littérature comparée et l'histoire littéraire à l'université de Haute-Alsace. Ses domaines et ses thèmes de recherche sont les suivants : la culture et la politique, la réception comparée et l'intermédialité (littérature, photographie et cinéma). 